\title{
Traveltime approximations for transversely isotropic media with an inhomogeneous background $^{a}$
}

${ }^{a}$ Published in Geophysics, 76, no. 3, WA31-WA42, (2011)

Tariq Alkhalifah ${ }^{1}$

\begin{abstract}
A transversely isotropic model with a tilted symmetry axis (TI) is regarded as one of the most effective approximations to the Earth subsurface, especially for imaging purposes. However, we commonly utilize this model by setting the axis of symmetry normal to the reflector. This assumption may be accurate in many places, but deviations from this assumption will cause errors in the wavefield description. Using perturbation theory and Taylor's series, I expand the solutions of the eikonal equation for $2 \mathrm{D}$ transversely isotropic media with respect to the independent parameter $\theta$, the angle the tilt of the axis of symmetry makes with the vertical, in a generally inhomogeneous TI background with a vertical axis of symmetry (VTI). I do an additional expansion in terms of the independent (anellipticity) parameter $\eta$ in a generally inhomogeneous elliptically anisotropic background medium. These new TI traveltime solutions are given by expansions in $\eta$ and $\theta$ with coefficients extracted from solving linear firstorder partial differential equations. Pade approximations are used to enhance the accuracy of the representation by predicting the behavior of the higher-order terms of the expansion. A simplification of the expansion for homogenous media provides nonhyperbolic moveout descriptions of the traveltime for TI models that are more accurate than other recently derived approximations. In addition, for 3D media, I develop traveltime approximations using Taylor's series type of expansions in the azimuth of the axis of symmetry. The coefficients of all these expansions can also provide us with the medium sensitivity gradients (Jacobian) for nonlinear tomographic-based inversion for the tilt in the symmetry axis.
\end{abstract}

\footnotetext{
${ }^{1}$ e-mail: tariq.alkhalifah@kaust.edu.sa
} 


\section{INTRODUCTION}

The nature of sedimentation and thin layering in the Earth subsurface induces wave propagation characteristics that can be better described by considering the medium to be anisotropic. Specifically, since the layering has a general preferred direction, we find that the transversely isotropic (TI) assumption to be the most practical type of anisotropy to represent big parts of the subsurface. The tilt in this case is naturally set in the direction normal to the layering (Alkhalifah and Bednar, 2000; Audebert et al., 2006; Behera and Tsvankin, 2009; Alkhalifah and Sava, 2010). Thus, this type of model approximates a big portion of the anisotropy resulting from the thin layering. Developing simple traveltime formulations for such a model helps in many applications, including traveltime tomography and integral-based Kirchhoff imaging. The vertical symmetry axis (VTI) medium is a special case of TI in which the symmetry axis is normal to the typically horizontal acquisition surface, and thus results in simpler formulations.

Traveltimes are conventionally evaluated by solving a nonlinear partial differential equation (PDE), better known as the eikonal equation. Among the most known methods for solving this equation are ray tracing and the finite-difference approximations. Finite-difference solutions of the eikonal equation have been recognized as one of the most efficient means of traveltime calculations (Vidale, 1990; van Trier and Symes, 1991; Popovici, 1991; Alkhalifah and Fomel, 2001). Some of main advantages of this method in comparison to ray tracing include the ability to directly provide solutions on regular grids, a complete coverage of the solution space, and a high numerical robustness. On the down side, finite-difference based solutions typically include only the first arrivals, which might not be even the most energetic ones (Cerveny, 2001). In anisotropic media, traveltime computation is dependent on more than one parameter field. However, through careful parametrization of the TI medium, $P$-wave traveltimes in 3D, under the acoustic assumption, become dependent on only three parameter fields and two angles. These parameters include the tilt-direction velocity, $v_{t}$, the normal-moveout equivalent velocity, $v=v_{t} \sqrt{1+2 \delta}$ [where $\delta$ corresponds to the symmetry direction (Tsvankin, 1997)], and the anellipticity parameter $\eta=\frac{\epsilon-\delta}{1+2 \delta}$ (with $\epsilon$ also defined with respect to the symmetry direction). This is evident in the eikonal equation for TI media developed by Alkhalifah $(1998,2000 \mathrm{a})$. If the symmetry axis is not vertical, two additional parameters are needed to describe the tilt in $3 \mathrm{D}$, the angle $\theta$ that the symmetry axis makes with the vertical and the azimuth $\phi$ of the vertical symmetry-axis plane with respect the $x$-axis [Tsvankin (1997)].

The process of finding a stable solution for the TI (or even the VTI) eikonal equation using finite-difference schemes is generally hard, especially since such a process requires finding the root of a quartic equation at each computational step (Wang et al., 2006). However, traveltime computation for a slightly more simplified, but not practical, elliptically anisotropic model is far more efficient. The reason for the high efficiency is that elliptical anisotropy has the same order of complexity (nonlinearity) in the eikonal equation as does the isotropic equation. Thus, though elliptical anisotropy represents an uncommon model in practice, it provides some flexibility in 
treating the difference between vertical and horizontal velocities, or in other words, the flexibility of stretching the depth axis to obtain accurate reflection depths in imaging (Peng and Steenson, 2001; Ball, 1995; Ohlsen and MacBeth, 1999). However, elliptical anisotropy does not provide accurate focusing for media of typical non-elliptical TI anisotropy (Alkhalifah and Larner, 1994). It will be used here however as the background medium for the perturbation expansions.

The forward problem, whether it is traveltime calculation or wavefield modeling, is a major component of the inversion process. If we model wavefields, we can use those wavefields to generate synthetic data and compare them with measured data as part of what we refer to as wavefield inversion. Likewise, forward traveltime calculation is used to measure the traveltime misfit with those extracted from the data in what is referred to as traveltime tomography. If we assume that one or two of the parameters are constant, the gradient of the objective function with respect to these parameters can be calculated analytically and that usually helps the inversion process. In this paper, I develop simplified formulations for traveltime calculation that can help in resolving anisotropy parameters, specifically the tilt angle.

Alkhalifah (2010) developed an eikonal-based scanning scheme to search for the anisotropy parameter $\eta$ that can provide the best traveltime fit to the data in a general inhomogeneous background medium. In this earlier paper, I derived first-order linear partial differential equations (PDEs) governing the coefficients of expanding the traveltime solution for VTI media in terms of the independent parameter $\eta$ from a background elliptical anisotropic model. I use the Shanks transform [Bender and Orszag (1978)] to enhance the accuracy of the expansion to a point in which the homogeneous-medium versions of it provided exceptional accuracy in describing the traveltime compared to other well-known published moveout equations (Alkhalifah, 2000b). I also suggested a simple angle transformation to make the method work for a tilted symmetry axis with a known tilt direction (i.e. in the direction normal to the layering). However, if the tilt direction is unknown, the $\eta$ estimation will certainly suffer from this limitation.

In this paper, I derive multi-parameter expansions of traveltime as a function of the symmetry-axis angles and $\eta$ with coefficients estimated using linearized forms of the eikonal equation. The accuracy of such an expansion is again further enhanced using Shanks transform and Pade approximations to obtain higher-order representation. I use a homogeneous-background medium version of the approximation to test its accuracy and then examine the 3D case where the axis direction is described by azimuth as well. 


\section{THE TI EIKONAL AND EXPANSION IN $\theta$}

In VTI media, the eikonal equation (Alkhalifah, 1998) in the acoustic approximation has the form:

$$
v^{2}(1+2 \eta)\left(\left(\frac{\partial \tau}{\partial x}\right)^{2}+\left(\frac{\partial \tau}{\partial y}\right)^{2}\right)+v_{t}^{2}\left(\frac{\partial \tau}{\partial z}\right)^{2}\left(1-2 \eta v^{2}\left(\left(\frac{\partial \tau}{\partial x}\right)^{2}+\left(\frac{\partial \tau}{\partial y}\right)^{2}\right)\right)=1
$$

where $\tau(x, y, z)$ is the traveltime (eikonal) measured from the source to a point with the coordinates $(x, y, z)$, and $v_{t}$ and $v$ are the velocity and NMO velocity $\left(=v_{t} \sqrt{1+2 \delta}\right)$, respectively, described with respect to the symmetry direction at that point. To formulate a well-posed initial-value problem for equation 1 , it is sufficient to specify $\tau$ at some closed surface and to choose one of the two solutions: the wave going from or toward the source. The level of nonlinearity in this quartic (in terms of $\tau$ ) equation is higher than that for the isotropic or elliptically anisotropic eikonal equations. This results in much more complicated finite-difference approximations of the VTI eikonal equation.

For a tilted TI medium, the traveltime derivatives in equation 1 are taken with respect to the tilt direction, and thus, we have to rotate the derivatives in equation 1 using the following Jacobian in 3D:

$$
\left(\begin{array}{ccc}
\cos \phi \cos \theta & \sin \phi \cos \theta & \sin \theta \\
-\sin \phi & \cos \phi & 0 \\
-\cos \phi \sin \theta & -\sin \phi \sin \theta & \cos \theta
\end{array}\right)
$$

to obtain an eikonal equation corresponding to the conventional computational coordinates governed by the acquisition surface. In equation $2, \theta$ is the angle of the symmetry axis measured from the vertical and $\phi$ corresponds to the azimuth of the vertical plane that contains the symmetry axis measured from the $x$-axis (the axis of the source-receiver direction). Setting initially $\phi=0$, for simplicity, allows us to obtain the eikonal equation for 2D TI media given by:

$$
\begin{aligned}
& v^{2}(1+2 \eta)\left(\cos \theta \frac{\partial \tau}{\partial x}+\sin \theta \frac{\partial \tau}{\partial z}\right)^{2}+ \\
& v_{t}^{2}\left(\cos \theta \frac{\partial \tau}{\partial z}-\sin \theta \frac{\partial \tau}{\partial x}\right)^{2}\left(1-2 \eta v^{2}\left(\cos \theta \frac{\partial \tau}{\partial x}+\sin \theta \frac{\partial \tau}{\partial z}\right)^{2}\right)=1
\end{aligned}
$$

The full 3D version of this equation is stated in Appendix D.

Solving equation 3 numerically requires solving a quartic equation (instead of the quadratic in the isotropic and elliptical anisotropic case) at each computational step. Alternatively, it can be solved using perturbation theory (Bender and Orszag, 1978) by approximating equation 3 with a series of simpler linear equations. Considering $\theta$ constant and small, we can represent the traveltime solution as a series expansion in $\theta$. This will result in a solution that is globally representative in the space domain 
Figure 1: A schematic plot showing the relation between a background traveltime field for $\theta=0$ and that when $\theta$ is larger than zero. The round dot at the top of the $\theta=0$ plane represents a source.

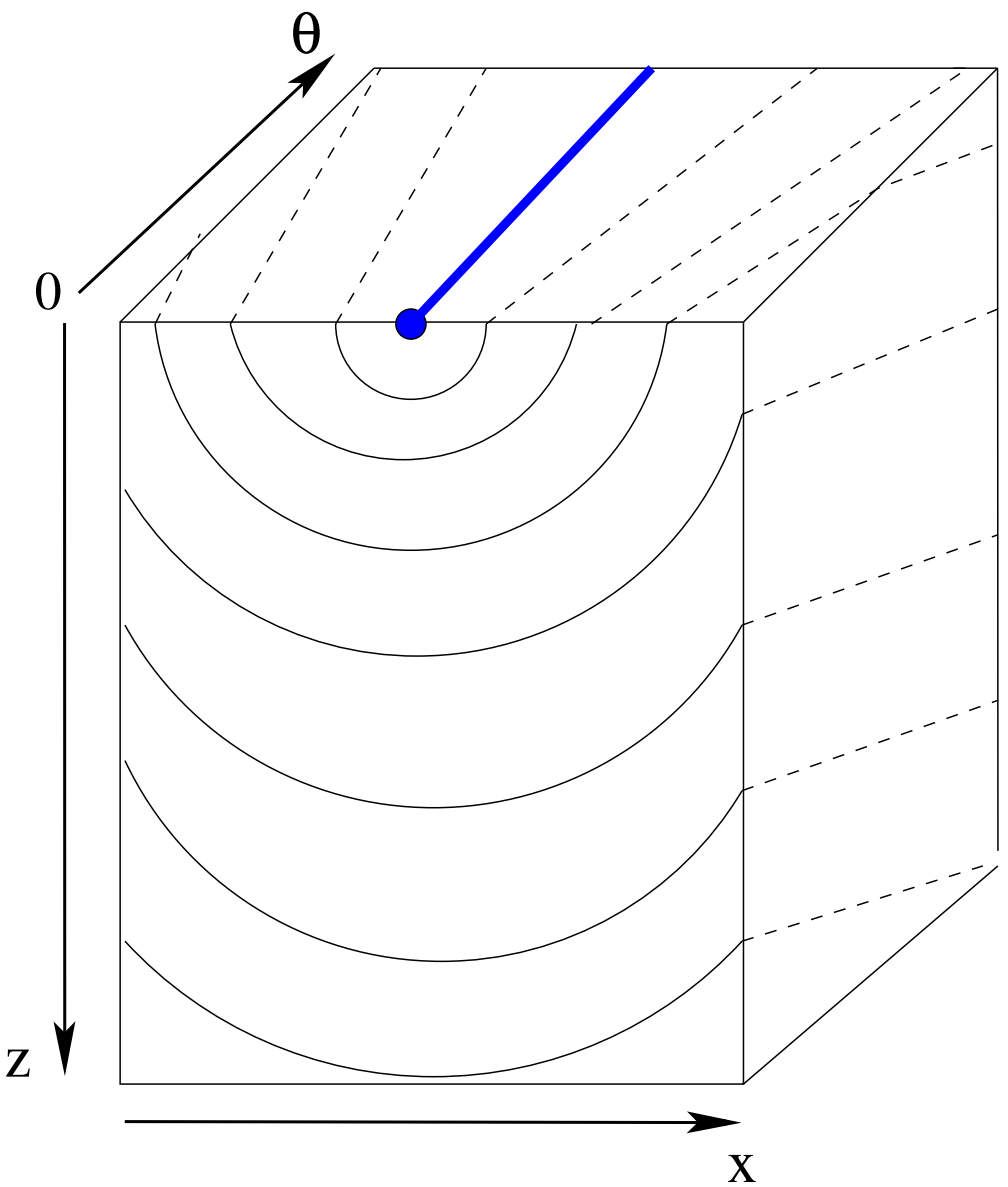


and, despite the approximation of small $\theta$, the accuracy for even large $\theta$, as we will see later, is high. The constant- $\theta$ assumption assumes a factorized medium (Alkhalifah, 1995) in $\theta$ (useful for smooth $\theta$ estimation applications). However, all other velocities and parameters including $v_{t}, v$ (or $\delta$ ) and $\eta$ are allowed to vary. Figure 1 illustrates the concept of the global expansion as we predict the traveltime for any $\theta$ from its behavior at $\theta=0$ for the full traveltime field using, in this case, a quadratic approximation. Specifically, we substitute the following trial solution,

$$
\tau(x, z) \approx \tau_{0}(x, z)+\tau_{1}(x, z) \sin \theta+\tau_{2}(x, z) \sin ^{2} \theta,
$$

where $\tau_{0}, \tau_{1}$, and $\tau_{2}$ are coefficients of the expansion with dimensions of traveltime, into the eikonal equation 3. For practical purposes, I consider here only three terms of the expansion. As a result (as shown in Appendix A), $\tau_{0}$ satisfies the eikonal equation for VTI anisotropy, while $\tau_{1}$ and $\tau_{2}$ satisfy linear first-order partial differential equations having the following general form (see Appendix A):

$$
v^{2}(1+2 \eta) \frac{\partial \tau_{0}}{\partial x} \frac{\partial \tau_{i}}{\partial x}+v_{t}^{2} \frac{\partial \tau_{0}}{\partial z} \frac{\partial \tau_{i}}{\partial z}-2 \eta v^{2} v_{t}^{2} \frac{\partial \tau_{0}}{\partial z} \frac{\partial \tau_{0}}{\partial x}\left(\frac{\partial \tau_{0}}{\partial z} \frac{\partial \tau_{i}}{\partial x}+\frac{\partial \tau_{0}}{\partial x} \frac{\partial \tau_{i}}{\partial z}\right)=f_{i}(x, z),
$$

with $i=1,2$. The functions $f_{i}(x, z)$ get more complicated for larger $i$ and depend on terms that can be evaluated only sequentially. Therefore, these linear partial differential equations must be solved in the order of increasing $i$ starting with $i=1$.

\section{EXPANSION IN TERMS OF $\theta$ AND $\eta$}

Though the expansion in terms of $\theta$ in the previous section allowed us to estimate traveltimes for a tilted symmetry axis, it also required that we solve the eikonal equation for a VTI medium, which is relatively challenging. For inversion purposes, it also required knowledge of $\eta$, which might not be possible in TI media using initially a VTI approximation, especially if the tilt is large. However, an expansion in $\eta$, in addition to $\theta$ (from their zero values), will result in an elliptically anisotropic background medium and it will allow us to search for both $\eta$ and $\theta$, simultaneously, considering that the elliptical anisotropy model is known.

The two-parameter expansion can be obtained by substituting the following trial solution:

$\tau(x, z) \approx \tau_{0}(x, z)+\tau_{\eta}(x, z) \eta+\tau_{\theta}(x, z) \sin \theta+\tau_{\eta_{2}}(x, z) \eta^{2}+\tau_{\eta \theta}(x, z) \eta \sin \theta+\tau_{\theta_{2}}(x, z) \sin ^{2} \theta$

into equation 3 resulting in linear first-order partial differential equations having the following general form:

$$
v_{t}^{2} \frac{\partial \tau_{0}}{\partial z} \frac{\partial \tau_{i}}{\partial z}+v^{2} \frac{\partial \tau_{0}}{\partial x} \frac{\partial \tau_{i}}{\partial x}=f_{i}(x, z)
$$

with $i=\eta, \theta, \eta_{2}, \eta \theta, \theta_{2}$, and $\tau_{0}$ satisfies the eikonal equation for an elliptical anisotropic background model. Again, the function $f_{i}(x, z)$ gets more complicated for $i$ corresponding to the second-order term and it depends on terms for the first order and 
background medium solutions. Therefore, these linear partial differential equations also must be solved in succession starting with $i=\eta$ and $i=\theta$. As soon as the $\tau_{\eta}$, and $\tau_{\eta_{2}}$ coefficients are evaluated, they can be used, as Alkhalifah (2010) showed, to estimate the traveltime using the first-sequence of Shanks transform (Bender and Orszag, 1978), and as shown in Appendix B, has the form:

$\tau(x, z) \approx \tau_{0}(x, z)+\tau_{\theta}(x, z) \sin \theta+\tau_{\theta_{2}}(x, z) \sin ^{2} \theta+\frac{\eta\left(\tau_{\eta}(x, z)+\tau_{\eta \theta}(x, z) \sin \theta\right)^{2}}{\tau_{\eta}(x, z)+\tau_{\eta \theta}(x, z) \sin \theta-\eta \tau_{\eta_{2}}(x, z)}$.

The $\theta$ expansion does not adapt well to the Shanks transform requirements for predicting the behavior of the higher-order terms in $\theta$. In this case, the second-order approximation in the $\theta$ expansion is sufficient.

For $\eta$ and $\theta$ scan applications, the coefficients $\left(\tau_{0}, \tau_{\eta}, \tau_{\theta}, \tau_{\eta_{2}}, \tau_{\eta \theta}\right.$, and $\left.\tau_{\theta_{2}}\right)$ need to be evaluated only once and can be used with equation 8 to search for the best traveltime fit to those traveltimes extracted from the data.

\section{A HOMOGENEOUS MODEL TEST}

Though the equations above are developed for a general inhomogeneous background medium, I examine their accuracy in representing TI traveltime and traveltime moveout in the homogeneous case. This is convenient since most parameter scan-type applications (i.e. semblance velocity analysis) are performed considering an effective homogeneous medium.

As shown in Appendix C, I use the simple traveltime relation for an elliptically anisotropic homogeneous background to recursively solve for the coefficients of the traveltime expansion in $\theta$ and $\eta$, and thus, obtain analytical representations for coefficients $\tau_{0}, \tau_{\theta}, \tau_{\eta}, \tau_{\theta_{2}}, \tau_{\eta_{2}}$, and $\tau_{\eta \theta}$. Setting $\eta=0$, to allow for a simplified presentation, I obtain an analytical representation of traveltime for tilted elliptical anisotropy given by

$$
\begin{array}{r}
\tau(x, z)=\sqrt{\frac{x^{2}}{v^{2}}+\frac{z^{2}}{v_{t}^{2}}}\left(1+\frac{\left(v_{t}^{2}-v^{2}\right) x z \sin \theta}{v^{2} z^{2}+v_{t}^{2} x^{2}}+\right. \\
\left.\frac{\sin ^{2} \theta\left(-v^{4} z^{4}+v^{2} v_{t}^{2}\left(x^{4}+z^{4}\right)-v_{t}^{4} x^{4}\right)}{2\left(v^{2} z^{2}+v_{t}^{2} x^{2}\right)^{2}}\right)
\end{array}
$$

where the source is located at $x=0$ and $z=0$.

This formula type (equation 9) basically represents a moveout equation for traveltime in TI media as a function of offset (or $x$ ) and can be compared with equations developed explicitly to represent the moveout in TI media. Pech et al. (2003) derived the exact quartic moveout coefficient (i.e., the fourth-order term of the Taylor series expansion for squared traveltime) for pure (nonconverted) reflections in arbitrarily anisotropic, heterogeneous media. They also linearized the P-wave quartic coefficient in the anisotropy parameters for homogeneous tilted TI media above a horizontal and 
dipping reflector. For a horizontal TI layer, the P-wave fourth-order Taylor series with linearized expressions for both the NMO velocity (Grechka and Tsvankin, 2000) and the quartic coefficient (Pech et al., 2003) is given by:

$$
t^{2}(X)=t_{0}^{2}+\frac{\left(1-2 \delta+2 \epsilon \sin ^{2} \theta-14(\epsilon-\delta) \sin ^{2} \theta \cos ^{2} \theta\right)}{v_{t}^{2}} X^{2}+A_{4} X^{4}
$$

where

$$
A_{4}=-\frac{2 \eta \cos ^{4} \theta}{v_{t}^{4} t_{0}^{2}}
$$

$t_{0}$ is the two-way zero-offset time and $X$ is the offset $(=2 x)$. For zero tilt, it reduces to

$$
t^{2}(X, \theta=0)=t_{0}^{2}+\frac{(1-2 \delta)}{v_{t}^{2}} X^{2}-\frac{2 \eta}{v_{t}^{4} t_{0}^{2}} X^{4},
$$

which reveals the additional approximation involved in this equation as even the second-order term is linearized with respect to $\delta$. Meanwhile, setting $\theta=0$ in equation 9 yields an accurate description of the second-order term of the moveout with the NMO velocity in the denominator instead.

Grechka and Pech (2006) suggested rewriting equation 10 in the following form:

$t^{2}(X)=t_{0}^{2}+\frac{\left(1-2 \delta+2 \epsilon \sin ^{2} \theta-14(\epsilon-\delta) \sin ^{2} \theta \cos ^{2} \theta\right)}{v_{t}^{2}} X^{2}+\frac{2 \eta A_{4}}{2 \eta-v^{2}(1+2 \eta) A_{4} X^{2}} X^{4}$,

which typically provides higher-order accuracy as the additional offset component in the denominator of the fourth-order term tends to predict the behavior at very large offsets.

Sena (1991) presented an anisotropic approximation of the group velocity as a function of symmetry angle. These velocities can serve to obtain moveout equations as well, and they are given by

$$
t(X)=\frac{\sqrt{X^{2}+4 z^{2}} \sqrt{2(\delta-\epsilon) \sin ^{4}(\theta-\psi)-2 \delta \sin ^{2}(\theta-\psi)+1}}{v_{t}},
$$

where the ray angle, $\psi$, satisfies

$$
\psi=\tan ^{-1}\left(\frac{X}{2 z}\right) .
$$

Using an elliptical anisotropic background model with axis-direction velocity equal to $2 \mathrm{~km} / \mathrm{s}$ velocity, $\delta=0.2$, and tilt angle $\theta=20^{\circ}$, I compare the traveltime errors of the moveout equations extracted from our eikonal based formulations with those that are used for pure moveout approximations, equations 13 and 14. For a reflector at 
Figure 2: The relative traveltime error as a function of offset for an elliptically anisotropic model with $v=2 \mathrm{~km} / \mathrm{s}, \delta=0.2, \theta=20^{\circ}$, and a reflector depth $z=2 \mathrm{~km}$ for the new expansion in $\theta$ (solid black curve), the nonhyperbolic moveout equation 10 (solid gray curve), and for the Sena approximation (dashed black curve). 
depth $z=2 \mathrm{~km}$, Figure 2 shows the percentage traveltime errors as a function of offset for the equations given above. Clearly, equations 13 and 14, given by the solid grey and dashed curves, respectively, are less accurate in describing the traveltime behavior overall than the new formula (solid black curve), equation 9. The moveout equations have faired well for the vertical direction but performed poorly for larger offset.

Moreover, for a more practical case of TI, in which $\eta=0.2$, the errors, as shown in Figure 3, are smaller overall for the new equation (solid black curve) than the other approximations. Despite the larger error of our approximation near vertical, the departure of the other approximations from the accurate value at large offset reflect their near-offset based expansion. In the new equation, the expansion is with respect to small tilt, and thus, has an unbiased representation of the traveltime with respect to offset. As a result, the accuracy of the new equation is higher over all.

Figure 3: The relative traveltime error as a function of offset for a model with $v=2$ $\mathrm{km} / \mathrm{s}, \eta=0.2, \theta=20^{\circ}$, and a reflector depth $z=2 \mathrm{~km}$ for the new expansion in $\theta$ (solid black curve), the nonhyperbolic moveout equation 10 (dashed gray curve), and 13 (solid gray curve) and for Sena's approximation (dashed black curve).

The analytical equations developed in this section were meant to test the accuracy of the perturbation theory approximations applied to the eikonal equation. Though they show high accuracy in representing the moveout, the general perturbation formulations are not meant to be only used as an alternative to other available 
equations derived for the homogeneous case. Their perturbation from a background inhomogeneous model allows us to predict traveltime in more complex media.

\section{THE VERTICAL DIRECTION}

The vertical direction in the conventional seismic experiment is critical as the depth mistie (or vertical velocity) and the NMO velocity are typically measured with respect to the vertical direction regardless of the tilt in the symmetry angle. Specifically, the vertical velocity is extracted from the well check shots (typically vertical) and the moveout velocity given by the second derivative of traveltime with respect to phase angle, is measured in the vertical direction $(x=0)$. Setting $x=0$ in equation 9 , and considering the two-way traveltime, $t=2 \tau$, yields the following relation for the traveltime in the vertical direction:

$$
t(x=0, z)=2 \frac{z}{v_{t}}\left(1-\frac{1}{2} \sin ^{2} \theta\left(1-\frac{v_{t}^{2}}{v^{2}}\right)\right),
$$

which includes terms related to the symmetry-axis direction. From Figure 2 we can see how well this approximation predicted the vertical traveltime. This equation is convenient to use along with well information to predict the velocities along the symmetry axis. Actually, for $\eta \neq 0$ (anelliptic TI media), applying Pade approximations (Bender and Orszag, 1978) to the $\eta$ and $\sin \theta$ expansions, yields the following relation for traveltime in the vertical direction:

$$
t(x=0, z)=2 \frac{z}{v_{t}} \frac{v^{2}}{v^{2}+\frac{1}{2} \sin ^{2} \theta\left(v^{2}-v_{t}^{2}\right)} .
$$

Note that this equation is independent of $\eta$, which implies that for small tilt angles, $\eta$ has practically no influence on the vertical traveltime. This fact helps us better construct the background elliptical anisotropy, as $\eta$ has little influence on this process for small tilt angles. Combine the above equations with those related to the NMO (stacking) velocity in the vertical direction:

$$
\frac{1}{t_{0} v_{n m o}^{2}}=\frac{1}{2} \frac{\partial^{2} t}{\partial x^{2}}(x=0, z)=\frac{v_{t}}{z} \frac{v^{2}+\frac{3}{2} \sin ^{2} \theta\left(v^{2}-v_{t}^{2}\right)}{v^{4}},
$$

where $t_{0}$ is given by equation 16. If we use Shanks transform again for higher-order accuracy, such an NMO velocity equation has the following form:

$$
\frac{1}{t_{0} v_{n m o}^{2}}=\frac{z}{v_{t}} \frac{8 v_{t}^{2} v^{4}-2\left(4 v^{2}-9 v_{t}^{2}\right)\left(v^{2}-v_{t}^{2}\right)^{2} \sin ^{4} \theta+24 v_{t}^{2}\left(v^{2}-v_{t}^{2}\right) v^{2} \sin ^{2} \theta}{z^{2}\left(v^{2}\left(\sin ^{2} \theta+2\right)-v_{t}^{2} \sin ^{2} \theta\right)^{3}},
$$

where $t_{0}$ is given by equation 18. Again, these equations are independent of $\eta$. The combination of equations 16 and 18 or equations 17 and 19 for a known tilt direction can be used to estimate the velocity along the symmetry axis as well as $\delta\left(=\frac{v^{2}-v_{t}^{2}}{2 v_{t}^{2}}\right)$. 
Specifically, the above formulations provide a mechanism to evaluate the symmetrydirection (anisotropy) parameters from measurements obtained using the conventional seismic experiment, vertical and stacking velocities.

Focusing on the performance of approximation 8 for small offsets allows us to predict the accuracy of equations 16 and 18 as they are derived from equation 8 . Figure 4 repeats the example of Figure 2 with a tilt of $5^{\circ}$ (a), $10^{\circ}(\mathrm{b})$, and $20^{\circ}$ (c), and a focus on small offsets (near vertical). Of course, errors increase with the increase in the tilt angle as all approximations are for small tilt angles from vertical. Though the vertical direction error in the case of the new equations is higher, the slope of the error is almost zero indicating that the new equation should provide a good estimation of the NMO velocity (extracted from the second derivative of traveltime with respect to offset). This feature is critical since the errors associated with the other approximations (i.e. Sena (1991)) for the NMO representation are large. This also explains the higher accuracy of the new equations at higher offsets as the error gradient is small. It is also clear from Figure 4 that using Pade approximations to predict the higher-order terms of the expansion in $\theta$ did not increase the accuracy much (the difference between solid and dashed black curves). This is also observed for anelliptic TI as we will see next.
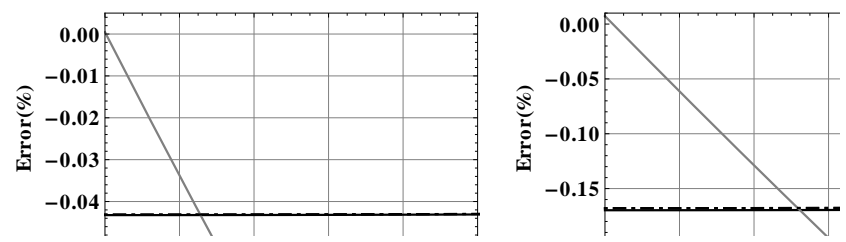

Figure 4: The relative traveltime error as a function of offset (near zero offset) for an elliptical anisotropic model with $v=2 \mathrm{~km} / \mathrm{s}, \delta=\epsilon=0.2, \theta=20^{\circ}$, and a reflector depth $z=2 \mathrm{~km}$ for the new expansion (equation 8) in $\theta$ (dashed black curve), the new expansion with Pade approximation in $\theta$ (solid black curve), and for the Sena approximation (solid gray curve). The plots correspond to (a) a 5 degree tilt, (b) a 10 degree tilt, and (c) a 20 degree tilt.

For anelliptic TI media with $\delta=0.1$ and $\epsilon=0.2$, I obtain similar results. Figure 5 shows the traveltime error over a limited offset for three symmetry tilt angles: (a) $5^{\circ}$, (b) $10^{\circ}$, and (c) $20^{\circ}$. Again, the accuracy of the new equations is apparent in the slope of the error near zero offset, implying that the NMO velocity representation is highly accurate. The errors in the vertical velocity, though, are the largest for the new equations. However, the vertical velocity genrally has less influence than the NMO velocity on time processing objectives.

Finally, for a TI medium with $\delta=0.0$ and $\epsilon=0.2$ (Figure 6) we observe similar 

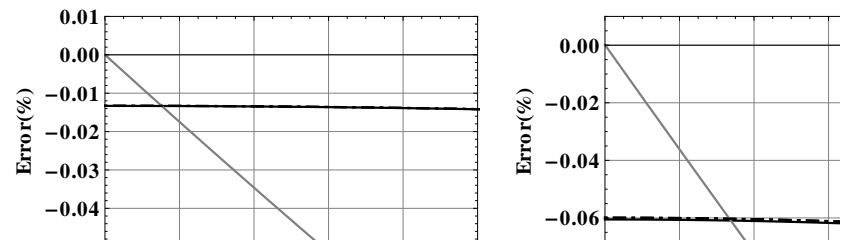

Figure 5: The relative traveltime error as a function of offset (near zero offset) for a TI model with $v=2 \mathrm{~km} / \mathrm{s}, \delta=0.1, \epsilon=0.2, \theta=20^{\circ}$, and a reflector depth $z=2 \mathrm{~km}$ for the new expansion (equation 8) in $\theta$ (dashed black curve), the new expansion with Pade approximation in $\theta$ (solid black curve), and for the Sena approximation (solid gray curve). The plots correspond to (a) a 5 degree tilt, (b) a 10 degree tilt, and (c) a 20 degree tilt.

behavior with smaller overall relative errors compared to Figures 4 and 5 . As $\delta$ decreases, the anisotropy influence near the vertical direction decreases, and the effect of the tilt is less pronounced. However, when the tilt is larger, Figure 6c, the errors are large and comparable to those in Figures $4 \mathrm{c}$ and $5 \mathrm{c}$ as the influence of $\eta$ starts to appear.
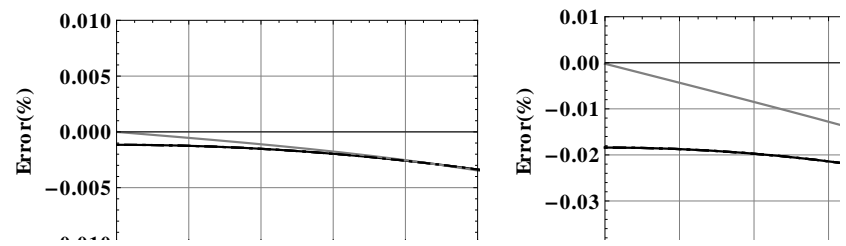

Figure 6: The traveltime error as a function of offset (near zero offset) for a TI anisotropic model with $v=2 \mathrm{~km} / \mathrm{s}, \delta=0.0, \epsilon=0.2, \theta=20^{\circ}$, and a reflector depth $z=2$ $\mathrm{km}$ for the new expansion (equation 8) in $\theta$ (dashed black curve), the new expansion with Pade approximation in $\theta$ (solid black curve), and for the Sina approximation (solid gray curve). The plots correspond to (a) a 5 degree tilt, (b) a 10 degree tilt, and (c) a 20 degree tilt.

In the above examples we note that despite the inferior accuracy of equations 16 and 18 in representing the vertical traveltime, with relative errors that could reach 0.3 percent for the 20 degree tilt case, it has superior qualities in predicting the NMO velocity. This phenomenon is explained by the fact that the new equations are 
expansions with respect to tilt angle, while the other equations are expansions with respect to offset (or anisotropy parameters), thus they provide better accuracy near zero offset. In contrast, the new equations tend to be more offset independent and better represent the moveout over larger offsets.

\section{THE SYMMETRY-AXIS AZIMUTH AND THE 3-D CASE}

In $3 \mathrm{D}$, the tilt of the symmetry axis is defined by an angle, $\theta$, measured from vertical, and the azimuth, $\phi$, of the vertical plane that contains the symmetry axis. Thus, $\phi$ is an angle measured in the horizontal plane from a given axis within that plane. To implement an expansion with respect to $\phi$, we must consider $\phi$ to be generally small. Since seismic acquisition is often performed in the dip direction of the structure, and we anticipate that the tilt is influenced by the presumed subsurface structure (folding), it would be reasonable to measure $\phi$ from the acquisition direction. In this case, I can consider $\phi$ to be small, and thus, approximate the traveltime solution of the eikonal equation with the following expansion:

$$
\tau(x, y, z) \approx \tau_{0}(x, y, z)+\tau_{1}(x, y, z) \sin \phi+\tau_{2}(x, y, z) \sin ^{2} \phi,
$$

to be inserted in the acoustic eikonal equation for TI in 3D given by

$$
a_{4} v_{t}^{4}\left(\frac{\partial \tau}{\partial z}\right)^{4}+a_{3} v_{t}^{3}\left(\frac{\partial \tau}{\partial z}\right)^{3}+a_{2} v_{t}^{2}\left(\frac{\partial \tau}{\partial z}\right)^{2}+a_{1} v_{t} \frac{\partial \tau}{\partial z}+a_{0}=0
$$

where $a_{0}, a_{1}, a_{2}, a_{3}$, and $a_{4}$ are stated in Appendix D. This is a complicated eikonal equation that is highly nonlinear, and thus, justifies our effort to simplify it through perturbation theory.

Substituting the trial solution, equation 20, into the eikonal equation for 3D TI media, equation 21, yields a polynomial expansion in the powers of $\sin \phi$. The zeroorder term of this polynomial represents the eikonal equation for TI media for the zero-azimuth case (the 2-D result). The coefficient of the $\sin \phi$ term yields a firstorder PDE for $\tau_{1}$. For simplicity, it is shown here for the case of elliptical anisotropy $(\eta=0)$ as

$$
\begin{aligned}
& \left(\left(v^{2} \cos ^{2} \theta+v_{t}^{2} \sin ^{2} \theta\right) \frac{\partial \tau_{0}}{\partial x}+\left(v^{2}-v_{t}^{2}\right) \sin \theta \cos \theta \frac{\partial \tau_{0}}{\partial z}\right) \frac{\partial \tau_{1}}{\partial x} \\
+ & v^{2} \frac{\partial \tau_{0}}{\partial y} \frac{\partial \tau_{1}}{\partial y} \\
+ & \left(\left(v^{2} \sin ^{2} \theta+v_{t}^{2} \cos ^{2} \theta\right) \frac{\partial \tau_{0}}{\partial z}+\left(v^{2}-v_{t}^{2}\right) \sin \theta \cos \theta \frac{\partial \tau_{0}}{\partial x}\right) \frac{\partial \tau_{1}}{\partial z} \\
= & \left(v^{2}-v_{t}^{2}\right) \sin \theta \frac{\partial \tau_{0}}{\partial y}\left(\cos \theta \frac{\partial \tau_{0}}{\partial z}-\sin \theta \frac{\partial \tau_{0}}{\partial x}\right) .
\end{aligned}
$$

We can obtain similar PDEs for the other coefficients of the expansion, but obtaining the $2 \mathrm{D}$ background TI model will be a challenging task. We can use the $2 \mathrm{D}$ version 
of the equation, developed earlier, to manage this task; however, the influence of ignoring the azimuth on that process could be critical.

A more realistic implementation is achieved by expanding from an elliptically anisotropic background with a vertical symmetry axis background in terms of $\eta, \theta$, and $\phi$, simultaneously. However, since a vertical symmetry axis has no particular azimuth (a singularity), I replace the tilt and azimuth by $n_{x}$ and $n_{y}\left(n_{x}=\sin \theta \cos \phi\right.$ and $\left.n_{y}=\sin \theta \sin \phi\right)$. For simplicity and to be able to include the resulting equations in this paper, I only consider first-order terms of the Taylor's series expansion, and thus, consider the following trial solution:

$$
\tau(x, y, z) \approx \tau_{0}(x, y, z)+\tau_{\eta}(x, y, z) \eta+\tau_{n_{x}}(x, y, z) n_{x}+\tau_{n_{y}}(x, y, z) n_{y} .
$$

In Appendix D, we look at higher-order expansions in $\eta$ to take advantage of the Shanks transform properties, which considerably helps in the $\eta$ case. Inserting equation 23 into equation 21 and equating terms of similar powers of the independent parameters $\left(\eta, n_{x}\right.$, and $\left.n_{y}\right)$ yields an elliptically anisotropic eikonal equation with a vertical symmetry axis from the zeroth-order term and first order PDEs from the other terms that have the same form as equation 7 . The source functions are shown in Appendix D.

Assuming a homogeneous-medium background yields an analytic relation, as shown in Appendix D [equation D-13]:

$$
\begin{aligned}
\tau(x, y, z) & \approx \sqrt{\frac{x^{2}}{v^{2}}+\frac{y^{2}}{v^{2}}+\frac{z^{2}}{v_{t}^{2}}}-\frac{v_{t}^{4} \eta\left(x^{2}+y^{2}\right)^{2} \sqrt{\frac{x^{2}+y^{2}}{v^{2}}+\frac{z^{2}}{v_{t}^{2}}}}{\left(v^{2} z^{2}+v_{t}^{2}\left(x^{2}+y^{2}\right)\right)^{2}} \\
& +\frac{\left(v_{t}^{2}-v^{2}\right) z \sin \theta}{v^{2} v_{t}^{2} \sqrt{\frac{x^{2}+y^{2}}{v^{2}}+\frac{z^{2}}{v_{t}^{2}}}}(x \cos \phi+y \sin \phi) .
\end{aligned}
$$

From this first-order approximation in all three parameters $\left(\eta, n_{x}\right.$, and $\left.n_{y}\right)$, we can see that by setting $v_{t}=v(\delta=0)$, the last term in the equation, that contains the tilt component, equals zero. This happens also if $\theta=0$ (axis is vertical), and the traveltime is dependent only on $\eta$. The $\eta$-term (second on the right hand side) in this first-order approximation has no dependence on symmetry angle. Also, note that this equation has a complex variation with offset (i.e. nonhyperbolic), however, reducing to a hyperbolic equation if $\eta$ and the symmetry angle are equal to zero.

\section{DISCUSSION}

The main objective of the newly developed expressions is parameter estimation in complex media. Specifically, the perturbation PDEs developed here are with respect to a background generally inhomogeneous, and possibly anisotropic, medium. If a generally inhomogeneous isotropic velocity field is available (for example from conventional migration velocity analysis), in addition to a map of the well-to-seismic 
misties, which can be used to develop a vertical velocity field, then an elliptical anisotropic model with a vertical symmetry axis can be constructed. We can use this model to solve for traveltimes in elliptically anisotropic media as a background model, as well as to solve for the expansion coefficients using equations 7 . These coefficients can be used with, for example, equation 23 to search explicitly for the $\eta$, and tilt angles $\theta$ and $\phi$ in 3D that provides the best traveltime fit to the data. This process can be implemented in a semblance-type search or incorporated as part of a tomographic inversion. Though the scans are based on an underline factorized assumption in the perturbation parameters, $\eta$ and the tilt angles, we can allow them to vary smoothly with location, and thus, produce effective values. The conversion of these effective values to interval ones in generally inhomogeneous media is not trivial and might require a tomographic treatment of its own.
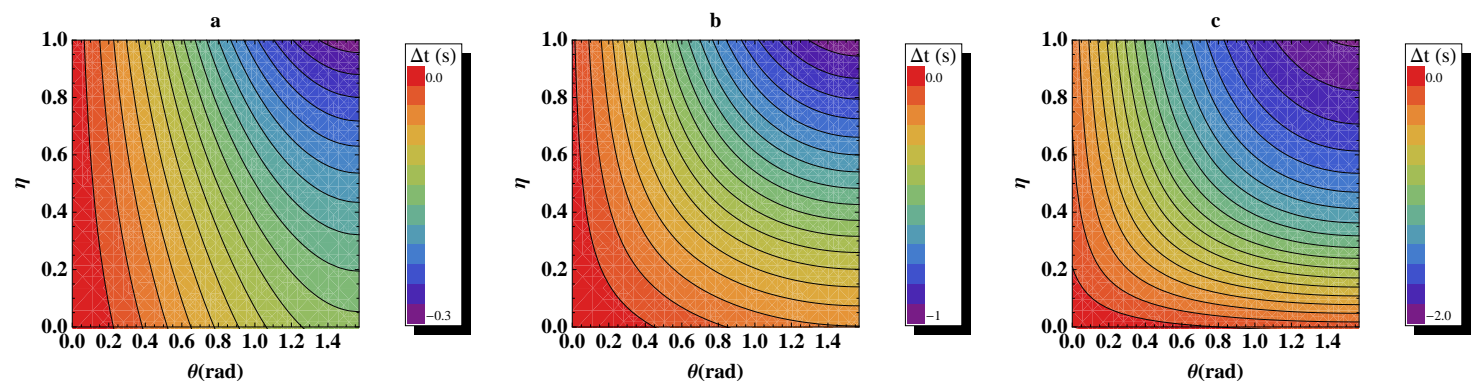

Figure 7: The traveltime difference between the TTI model computed using equation 9 and the elliptically anisotropic with a vertical symmetry axis background model for (a) an offset of $1 \mathrm{~km},(\mathrm{~b})$ an offset of $2 \mathrm{~km}$, and (c) an offset of $4 \mathrm{~km}$. The medium has $v_{t}=2 \mathrm{~km} / \mathrm{s}, v=2 \mathrm{~km} / \mathrm{s}(\delta=0)$, and a reflector depth, $z=2 \mathrm{~km}$.

In 3D, the search for $\eta$ and the symmetry direction angles can be applied either sequentially or to all the parameters at once. A sequential search, though faster and easier, may propagate some of the errors of an initial (wrong) tilt into the estimation of the parameter $\eta$ (Behera and Tsvankin, 2009). The search for all three parameters simultaneously would reduce such errors, but it will suffer from a null space based on the tradeoff between $\eta$ and the tilt angles. Conventionally, the information for $\eta$ could be extracted, especially for small tilt angles from vertical, which is assumed here, from long offsets and dipping reflectors. The tilt information resolution in 3D requires a 3D coverage (i.e. wide azimuth or even narrow azimuth for small tilt azimuth). There is also a general tradeoff, even in $2 \mathrm{D}$ media, between $\eta$ and the tilt angle, which may require some a priori information for $\eta$ or constraining the tilt angle to be normal to the reflector dip (Alkhalifah and Bednar, 2000). Figure 7 shows the dependence of traveltime in the $2 \mathrm{D}$ case, based on equation 9 , on the parameters $\eta$ and $\theta$. For a single offset, clearly there are combinations of $\eta$ and $\theta$ (given by the contour lines) that provide equal traveltimes. Nevertheless these curves clearly vary from one offset to another. Specifically, near offsets (Figure 7a, where $x / z=0.5$ ), for mostly small tilt angles, show little dependence of traveltime on $\eta$, and more 
dependence on $\theta$. On the other hand, as the tilt angle increases the resolution of $\eta$ increases as its influence starts to affect even the shorter offsets. The dependence of the traveltime on $\eta$ increases for $x / z=1$ (Figure $7 \mathrm{~b}$ ) and $x / z=2$ (Figure $7 \mathrm{c}$ ), which implies that $\eta$ is better resolved at large offsets for small tilt angles. However, it is resolved even better for large tilt angles in all cases. Meanwhile, larger offsets with reasonable $\eta$ values result in less dependence of the traveltime on tilt angle.

The availability of multi-offset data will increase our chances in resolving both $\eta$ and the tilt angle in 2D. The addition of multi azimuth should help resolve the tilt in 3D. Of course, the accuracy of resolving these parameters will depend mainly on how well we estimate the original elliptically anisotropic background medium. However, we can always go back and improve on our velocity picks once an approximate effective $\eta$ and tilt-angle fields are estimated. There are probably many other more sophisticated ways to explore this parameter matrix, however, the equations introduced here provides the basis for doing so.

\section{CONCLUSIONS}

Expanding the traveltime solutions of the TI eikonal equation in a power series in terms of independent parameters, like the tilt angle $\theta$ in 2D, provides an efficient tool to estimate $\theta$ in a generally inhomogeneous background medium. Additional expansions from a background elliptical anisotropic medium with a vertical symmetry axis allows us to search for the anisotropy parameter $\eta$ and the tilt angles simultaneously, even in 3D media. For a homogeneous background, I obtain analytic nonhyperbolic moveout equations for anisotropic media that are generally simple, and yet accurate. Nevertheless, the formulations provide $\eta$ and tilt-angles estimation capabilities for a general inhomogeneous background medium.

\section{ACKNOWLEDGMENTS}

I am grateful to KAUST for its financial support. I also thank Andrej Bona, Ilya Tsvankin, Ivan Psencik, and Andres Pech for their critical and helpful reviews of the paper.

\section{REFERENCES}

Alkhalifah, T., 1995, Efficient syntheticseismogram generation in transversely isotropic, inhomogeneous media: Geophysics, 60, 1139-1150.

— 1998 , Acoustic approximations for processing in transversely isotropic media: Geophysics, 63, 623-631.

—, $2000 \mathrm{a}$, An acoustic wave equation for anisotropic media: Geophysics, 65, $1239-1250$. 
, 2000b, The offset-midpoint traveltime pyramid in transversely isotropic media: Geophysics, 65, 1316-1325.

—_ 2010, Scanning anisotropy parameters in general inhomogeneous media: submitted to Geophysics.

Alkhalifah, T., and J. Bednar, 2000, Building a 3-D anisotropic model: Its implications to traveltime calculation and velocity analysis: 70th Ann. Internat. Mtg, Soc. of Expl. Geophys., 965-968.

Alkhalifah, T., and S. Fomel, 2001, Implementing the fast marching eikonal solver: spherical versus Cartesian coordinates: Geophys. Prosp., 49, 165-178.

Alkhalifah, T., and K. Larner, 1994, Migration error in transversely isotropic media: Geophysics, 59, 1405-1418.

Alkhalifah, T., and P. Sava, 2010, A transversely isotropic medium with a tilted symmetry axis normal to the reflector: Geophysics, 75, A19-A24.

Audebert, F. S., A. Pettenati, and V. Dirks, 2006, TTI anisotropic depth migration - which tilt estimate should we use?: EAGE, Expanded Abstracts, P185.

Ball, G., 1995, Estimation of anisotropy and anisotropic 3-D prestack depth migration, offshore Zaire: Geophysics, 60, 1495-1513.

Behera, L., and I. Tsvankin, 2009, Migration velocity analysis for tilted transversely isotropic media: Geophysical Prospecting, 57, 13-26.

Bender, C. M., and S. A. Orszag, 1978, Advanced mathematical methods for scientists and engineers: McGraw-Hill.

Cerveny, V., 2001, Seismic ray theory: Cambridge University Press.

Grechka, V., and A. Pech, 2006, Quartic reflection moveout in a weakly anisotropic dipping layer: Geophysics, 71, no. 1, D1-D13.

Grechka, V., and I. Tsvankin, 2000, Inversion of azimuthally dependent $\mathrm{NmO}$ velocity in transversely isotropic media with a tilted axis of symmetry: Geophysics, 65, 232-246.

Ohlsen, F., and C. MacBeth, 1999, Elliptical anisotropy: Regression or advance?: SEG, Expanded Abstracts, 18, 1600-1603.

Pech, A., I. Tsvankin, and V. Grechka, 2003, Quartic moveout coefficient: 3D description and application to tilted Ti media: Geophysics, 68, 1600-1610.

Peng, C., and K. Steenson, 2001, 3-D prestack depth migration in anisotropic media: A case study at the Lodgepole reef play in North Dakota: The Leading Edge, 20, $524-527$.

Popovici, M., 1991, Finite difference travel time maps, in SEP-70: Stanford Exploration Project, 245-256.

Sena, A. G., 1991, Seismic traveltime equations for azimuthally anisotropic and isotropic media: Estimation of interval elastic properties: Geophysics, 56, 20902101.

Tsvankin, I., 1997, Moveout analysis for tranversely isotropic media with a tilted symmetry axis: Geophysical Prospecting, 45, 479-512.

van Trier, J., and W. W. Symes, 1991, Upwind finite-difference calculation of traveltimes: Geophysics, 56, 812-821.

Vidale, J. E., 1990, Finite-difference calculation of traveltimes in three dimensions: Geophysics, 55, 521-526. 
Wang, Y., T. Nemeth, and R. T. Langan, 2006, An expanding-wavefront method for solving the eikonal equations in general anisotropic media: Geophysics, 71, T129-T135.

\section{APPENDIX A: EXPANSION IN $\theta$}

To derive a traveltime equation in terms of perturbations in $\theta$, we first establish the form for the governing equation for TI media given by the eikonal representation. The eikonal equation for $p$-waves in TI media in 2D (for simplicity) is given by

$$
\begin{aligned}
& v^{2}(1+2 \eta)\left(\cos \theta \frac{\partial \tau}{\partial x}+\sin \theta \frac{\partial \tau}{\partial z}\right)^{2}+ \\
& v_{t}^{2}\left(\cos \theta \frac{\partial \tau}{\partial z}-\sin \theta \frac{\partial \tau}{\partial x}\right)^{2}\left(1-2 \eta v^{2}\left(\cos \theta \frac{\partial \tau}{\partial x}+\sin \theta \frac{\partial \tau}{\partial z}\right)^{2}\right)=1
\end{aligned}
$$

To solve equation A-1 through perturbation theory, we assume that $\theta$ is small, and thus, a trial solution can be expressed as a series expansion in $\sin \theta$ given by

$$
\tau(x, z) \approx \tau_{0}(x, z)+\tau_{1}(x, z) \sin \theta+\tau_{2}(x, z) \sin ^{2} \theta
$$

where $\tau_{0}, \tau_{1}$ and $\tau_{2}$ are coefficients of the expansion given in units of traveltime, and, for practicality, terminated at the second power of $\sin \theta$. Inserting the trial solution, equation A-2, into equation A-1 yields a long formula, but by setting $\sin \theta=0, \mathrm{I}$ obtain the zeroth-order term given by

$$
v^{2}(1+2 \eta)\left(\frac{\partial \tau_{0}}{\partial x}\right)^{2}+v_{t}^{2}\left(\frac{\partial \tau_{0}}{\partial z}\right)^{2}\left(1-2 \eta v^{2}\left(\frac{\partial \tau_{0}}{\partial x}\right)^{2}\right)=1
$$

which is the eikonal formula for VTI anisotropy. By equating the coefficients of the powers of the independent parameter $\sin \theta$, in succession, we end up first with the coefficients of first-power in $\sin \theta$, simplified by using equation A-3, and given by

$$
\begin{gathered}
v^{2} \frac{\partial \tau_{1}}{\partial x}\left((2 \eta+1) \frac{\partial \tau_{0}}{\partial x}-2 v_{t}^{2} \eta \frac{\partial \tau_{0}}{\partial x}\left(\frac{\partial \tau_{0}}{\partial z}\right)^{2}\right)+v_{t}^{2} \frac{\partial \tau_{1}}{\partial z}\left(\frac{\partial \tau_{0}}{\partial z}-2 v^{2} \eta\left(\frac{\partial \tau_{0}}{\partial x}\right)^{2} \frac{\partial \tau_{0}}{\partial z}\right)= \\
2 v^{2} v_{t}^{2} \eta\left(\frac{\partial \tau_{0}}{\partial x}\left(\frac{\partial \tau_{0}}{\partial z}\right)^{3}-\left(\frac{\partial \tau_{0}}{\partial x}\right)^{3} \frac{\partial \tau_{0}}{\partial z}\right)-v^{2}(2 \eta+1) \frac{\partial \tau_{0}}{\partial x} \frac{\partial \tau_{0}}{\partial z}+v_{t}^{2} \frac{\partial \tau_{0}}{\partial x} \frac{\partial \tau_{0}}{\partial z}(\mathrm{~A}-4)
\end{gathered}
$$

which is a first-order linear partial differential equation in $\tau_{1}$. The coefficient of $\sin \theta^{2}$, with some manipulation, has the following form

$$
2 v^{2} \frac{\partial \tau_{2}}{\partial x}\left((2 \eta+1) \frac{\partial \tau_{0}}{\partial x}-2 v_{t}^{2} \eta \frac{\partial \tau_{0}}{\partial x}\left(\frac{\partial \tau_{0}}{\partial z}\right)^{2}\right)+2 v_{t}^{2} \frac{\partial \tau_{2}}{\partial z}\left(\frac{\partial \tau_{0}}{\partial z}-2 v^{2} \eta\left(\frac{\partial \tau_{0}}{\partial x}\right)^{2} \frac{\partial \tau_{0}}{\partial z}\right)=
$$




$$
\begin{gathered}
v^{2}(2 \eta+1)\left(\frac{\partial \tau_{0}}{\partial x}\right)^{2}+2 v^{2} v_{t}^{2} \eta\left(\frac{\partial \tau_{0}}{\partial z}\right)^{4}+2 v_{t}^{2} \frac{\partial \tau_{1}}{\partial x} \frac{\partial \tau_{0}}{\partial z}-v_{t}^{2}\left(\frac{\partial \tau_{1}}{\partial z}-\frac{\partial \tau_{0}}{\partial x}\right)^{2}+v_{t}^{2}\left(\frac{\partial \tau_{0}}{\partial z}\right)^{2} \\
+4 v^{2} v_{t}^{2} \eta \frac{\partial \tau_{1}}{\partial x}\left(\frac{\partial \tau_{0}}{\partial z}\right)^{3}-12 v^{2} v_{t}^{2} \eta\left(\frac{\partial \tau_{0}}{\partial x}\right)^{2}\left(\frac{\partial \tau_{0}}{\partial z}\right)^{2}+2 v^{2} v_{t}^{2} \eta\left(\frac{\partial \tau_{1}}{\partial x}\right)^{2}\left(\frac{\partial \tau_{0}}{\partial z}\right)^{2} \\
+12 v^{2} v_{t}^{2} \eta \frac{\partial \tau_{0}}{\partial x} \frac{\partial \tau_{1}}{\partial z}\left(\frac{\partial \tau_{0}}{\partial z}\right)^{2}-12 v^{2} v_{t}^{2} \eta\left(\frac{\partial \tau_{0}}{\partial x}\right)^{2} \frac{\partial \tau_{1}}{\partial x} \frac{\partial \tau_{0}}{\partial z}+8 v^{2} v_{t}^{2} \eta \frac{\partial \tau_{0}}{\partial x} \frac{\partial \tau_{1}}{\partial x} \frac{\partial \tau_{1}}{\partial z} \frac{\partial \tau_{0}}{\partial z} \\
-v^{2}(2 \eta+1)\left(\frac{\partial \tau_{1}}{\partial x}+\frac{\partial \tau_{0}}{\partial z}\right)^{2}+2 v^{2} v_{t}^{2} \eta\left(\frac{\partial \tau_{0}}{\partial x}\right)^{2}\left(\frac{\partial \tau_{1}}{\partial z}-\frac{\partial \tau_{0}}{\partial x}\right)^{2}-2 v^{2}(2 \eta+1) \frac{\partial \tau_{0}}{\partial x} \frac{\partial \tau_{1}}{\partial z}(\mathrm{~A}-5)
\end{gathered}
$$

which is again a first-order linear partial differential equation in $\tau_{2}$ with an obviously more complicated source function given by the right-hand side. Though the equation seems complicated, many of the variables of the source function (right-hand side) can be evaluated during the evaluation of equations A-3 and A-4 in a fashion that will not add much to the cost.

\section{APPENDIX B: EXPANSION IN $\theta$ AND $\eta$}

For an expansion in $\theta$ and $\eta$, simultaneously, I use the following trial solution:

$\tau(x, z) \approx \tau_{0}(x, z)+\tau_{\eta}(x, z) \eta+\tau_{\theta}(x, z) \sin \theta+\tau_{\eta_{2}}(x, z) \eta^{2}+\tau_{\eta \theta}(x, z) \eta \sin \theta+\tau_{\theta_{2}}(x, z) \sin ^{2} \theta$,

in terms of the coefficients $\tau_{i}$, where the $i$ corresponds to $\eta, \theta, \eta_{2}, \eta \theta$, and $\theta_{2}$. Inserting the trial solution, equation B-1, into equation A-1 yields again a long formula, but by setting both $\sin \theta=0$ and $\eta=0$, I obtain the zeroth-order term given by

$$
v^{2}(x, y, z)\left(\frac{\partial \tau_{0}}{\partial x}\right)^{2}+v_{t}^{2}(x, y, z)\left(\frac{\partial \tau_{0}}{\partial z}\right)^{2}=1
$$

which is simply the eikonal formula for elliptical anisotropy. By equating the coefficients of the powers of the independent parameter $\sin \theta$ and $\eta$, in succession starting with first powers of the two parameters, we end up first with the coefficients of firstpower in $\sin \theta$ and zeroth power in $\eta$, simplified by using equation B-2, and given by

$$
v^{2} \frac{\partial \tau_{0}}{\partial x} \frac{\partial \tau_{\theta}}{\partial x}+v_{t}^{2} \frac{\partial \tau_{0}}{\partial z} \frac{\partial \tau_{\theta}}{\partial z}=-\left(v^{2}-v_{t}^{2}\right) \frac{\partial \tau_{0}}{\partial x} \frac{\partial \tau_{0}}{\partial z}
$$

which is a first-order linear partial differential equation in $\tau_{\theta}$. The coefficients of zero-power in $\sin \theta$ and the first-power in $\eta$ is given by

$$
v^{2} \frac{\partial \tau_{0}}{\partial x} \frac{\partial \tau_{\eta}}{\partial x}+v_{t}^{2} \frac{\partial \tau_{0}}{\partial z} \frac{\partial \tau_{\eta}}{\partial z}=-\left(v^{2}\left(\frac{\partial \tau_{0}}{\partial x}\right)^{2}-v^{2} v_{t}^{2}\left(\frac{\partial \tau_{0}}{\partial x}\right)^{2}\left(\frac{\partial \tau_{0}}{\partial z}\right)^{2}\right)
$$

The coefficients of the square terms in $\sin \theta$, with some manipulation, results in the following relation

$$
2 v^{2} \frac{\partial \tau_{0}}{\partial x} \frac{\partial \tau_{\theta_{2}}}{\partial x}+2 v_{t}^{2} \frac{\partial \tau_{0}}{\partial z} \frac{\partial \tau_{\theta_{2}}}{\partial z}=v^{2}\left(\frac{\partial \tau_{0}}{\partial x}\right)^{2}-v^{2}\left(\frac{\partial \tau_{\theta}}{\partial x}\right)^{2}-
$$




$$
\begin{gathered}
2\left(v^{2}-v_{t}^{2}\right) \frac{\partial \tau_{\theta}}{\partial x} \frac{\partial \tau_{0}}{\partial z}-2\left(v^{2}-v_{t}^{2}\right) \frac{\partial \tau_{0}}{\partial x} \frac{\partial \tau_{\theta}}{\partial z}- \\
v_{t}^{2}\left(\frac{\partial \tau_{0}}{\partial x}\right)^{2}-v_{t}^{2}\left(\frac{\partial \tau_{\theta}}{\partial z}\right)^{2}-\left(v^{2}-v_{t}^{2}\right)\left(\frac{\partial \tau_{0}}{\partial z}\right)^{2}
\end{gathered}
$$

which is again a first-order linear partial differential equation in $\tau_{\theta_{2}}$ with an obviously more complicated source function given by the right hand side. The coefficients of the square terms in $\eta$, with also some manipulation, results in the following relation

$$
\begin{aligned}
2 v^{2} \frac{\partial \tau_{0}}{\partial x} \frac{\partial \tau_{\eta_{2}}}{\partial x}+2 v_{t}^{2} \frac{\partial \tau_{0}}{\partial z} \frac{\partial \tau_{\eta_{2}}}{\partial z}= & 4 v_{t}^{2} v^{2} \frac{\partial \tau_{0}}{\partial x} \frac{\partial \tau_{0}}{\partial z}\left(\frac{\partial \tau_{\eta}}{\partial x} \frac{\partial \tau_{0}}{\partial z}+\frac{\partial \tau_{0}}{\partial x} \frac{\partial \tau_{\eta}}{\partial z}\right)- \\
& v^{2}\left(\frac{\partial \tau_{\eta}}{\partial x}\right)^{2}-4 v^{2} \frac{\partial \tau_{0}}{\partial x} \frac{\partial \tau_{\eta}}{\partial x}-v_{t}^{2}\left(\frac{\partial \tau_{\eta}}{\partial z}\right)^{2}
\end{aligned}
$$

which is again a first-order linear partial differential equation in $\tau_{\eta_{2}}$ with a again complicated source function.

Finally, the coefficients of the first-power terms in both $\sin \theta$ and $\eta$ results also in a first-order linear partial differential equation in $\tau_{\eta \theta}$ given by

$$
\begin{aligned}
& 2 v^{2} \frac{\partial \tau_{0}}{\partial x} \frac{\partial \tau_{\eta \theta}}{\partial x}+2 v_{t}^{2} \frac{\partial \tau_{0}}{\partial z} \frac{\partial \tau_{\eta \theta}}{\partial z}= \\
& 4 v_{t}^{2} v^{2} \frac{\partial \tau_{0}}{\partial x} \frac{\partial \tau_{0}}{\partial z}\left(\frac{\partial \tau_{0}}{\partial z}\left(\frac{\partial \tau_{\theta}}{\partial x}+\frac{\partial \tau_{0}}{\partial z}\right)+\frac{\partial \tau_{0}}{\partial x}\left(\frac{\partial \tau_{\theta}}{\partial z}-\frac{\partial \tau_{0}}{\partial x}\right)\right) \\
& -2 v^{2} \frac{\partial \tau_{0}}{\partial x} \frac{\partial \tau_{\eta}}{\partial z}-4 v^{2} \frac{\partial \tau_{0}}{\partial x}\left(\frac{\partial \tau_{\theta}}{\partial x}+\frac{\partial \tau_{0}}{\partial z}\right)-2 v^{2} \frac{\partial \tau_{\eta}}{\partial x}\left(\frac{\partial \tau_{\theta}}{\partial x}+\frac{\partial \tau_{0}}{\partial z}\right) \\
& +2 v_{t}^{2} \frac{\partial \tau_{\eta}}{\partial x} \frac{\partial \tau_{0}}{\partial z}-2 v_{t}^{2} \frac{\partial \tau_{\eta}}{\partial z}\left(\frac{\partial \tau_{\theta}}{\partial z}-\frac{\partial \tau_{0}}{\partial x}\right) .
\end{aligned}
$$

Though the equation seems complicated, many of the variables of the source function (right hand side) can be evaluated during the evaluation of equations B-3 and B-4 in a fashion that will not add much to the cost.

Using Shanks transforms (Bender and Orszag, 1978) we can isolate and remove the most transient behavior of the expansion B- 1 in $\eta$ (the $\theta$ expansion did not improve with such a treatment) by first defining the following parameters:

$$
\begin{aligned}
& A_{0}=\tau_{0}+\tau_{\theta} \sin \theta+\tau_{\theta_{2}} \sin ^{2} \theta \\
& A_{1}=A_{0}+\left(\tau_{\eta}+\tau_{\eta \theta} \sin \theta\right) \eta \\
& A_{2}=A_{1}+\tau_{\eta_{2}} \eta^{2}
\end{aligned}
$$

The first sequence of Shanks transforms uses $A_{0}, A_{1}$, and $A_{2}$, and thus, is given by

$$
\begin{aligned}
\tau(x, z) \approx \frac{A_{0} A_{2}-A_{1}^{2}}{A_{0}-2 A_{1}+A_{2}}= & \tau_{0}(x, z)+\tau_{\theta}(x, z) \sin \theta+\tau_{\theta_{2}}(x, z) \sin ^{2} \theta \\
& +\frac{\eta\left(\tau_{\eta}(x, z)+\tau_{\eta \theta}(x, z) \sin \theta\right)^{2}}{\tau_{\eta}(x, z)+\tau_{\eta \theta}(x, z) \sin \theta-\eta \tau_{\eta_{2}}(x, z)} .
\end{aligned}
$$




\section{APPENDIX C: THE HOMOGENEOUS MEDIUM CASE}

To develop analytical traveltime representation for TI media, I start with a background velocity model that is homogeneous. The expansion here will be with respect to $\eta$ and $\theta$ from a background elliptical anisotropic model. In this case, the traveltime from a point source at $x=0$ and $z=0$ is given by the following simple relation in 2-D:

$$
\tau_{0}(x, z)=\sqrt{\frac{x^{2}}{v^{2}}+\frac{z^{2}}{v_{t}^{2}}}
$$

which satisfies the eikonal equation B-2. Using equation C-1, I evaluate $\frac{\partial \tau_{0}}{\partial x}$ and $\frac{\partial \tau_{0}}{\partial z}$ and insert them into equation B-3 to solve the first-order linear equation to obtain

$$
\tau_{\theta}(x, z)=\frac{\left(v_{t}^{2}-v^{2}\right) x z \sqrt{\frac{x^{2}}{v^{2}}+\frac{z^{2}}{v_{t}^{2}}}}{v^{2} z^{2}+v_{t}^{2} x^{2}}
$$

as well as insert them into equation B-4 and solve the equation to obtain

$$
\tau_{\eta}(x, z)=-\frac{v_{t}^{4} x^{4} \sqrt{\frac{x^{2}}{v^{2}}+\frac{z^{2}}{v_{t}^{2}}}}{\left(v^{2} z^{2}+v_{t}^{2} x^{2}\right)^{2}}
$$

I now evaluate $\frac{\partial \tau_{\theta}}{\partial x}$ and $\frac{\partial \tau_{\theta}}{\partial z}$ and use them to solve equation B-5. After some tedious algebra, I obtain

$$
\tau_{\theta_{2}}(x, z)=\frac{\sqrt{\frac{x^{2}}{v^{2}}+\frac{z^{2}}{v_{t}^{2}}}\left(-v^{4} z^{4}+v^{2} v_{t}^{2}\left(x^{4}+z^{4}\right)-v_{t}^{4} x^{4}\right)}{2\left(v^{2} z^{2}+v_{t}^{2} x^{2}\right)^{2}} .
$$

I also evaluate $\frac{\partial \tau_{\eta}}{\partial x}$ and $\frac{\partial \tau_{\eta}}{\partial z}$ and use them to solve equation B-6 to obtain

$$
\tau_{\eta_{2}}(x, z)=\frac{3 v_{t}^{6} x^{6} \sqrt{\frac{x^{2}}{v^{2}}+\frac{z^{2}}{v_{t}^{2}}}\left(4 v^{2} z^{2}+v_{t}^{2} x^{2}\right)}{2\left(v^{2} z^{2}+v_{t}^{2} x^{2}\right)^{4}} .
$$

Finally, I solve equation B-7. After some tedious algebra once again, I obtain

$$
\tau_{\eta \theta}(x, z)=-\frac{v_{t}^{4} x^{3} z \sqrt{\frac{x^{2}}{v^{2}}+\frac{z^{2}}{v_{t}^{2}}}\left(\left(3 v^{2}+v_{t}^{2}\right) x^{2}+4 v^{2} z^{2}\right)}{\left(v^{2} z^{2}+v_{t}^{2} x^{2}\right)^{3}} .
$$

Using the first sequence of Shanks transform, equation B-9, applied to the Taylor's series expansion, we obtain an analytical equation that describes traveltime as a function of $\eta$ and $\theta$.

For 3-D media, I include the azimuth angle as we will see next. 


\section{APPENDIX D: EXPANSION IN 3D}

The eikonal equation for $P$-waves in a TI medium with a tilt in the symmetry axis satisfies the following relation,

$$
a_{4} v_{t}^{4}\left(\frac{\partial \tau}{\partial z}\right)^{4}+a_{3} v_{t}^{3}\left(\frac{\partial \tau}{\partial z}\right)^{3}+a_{2} v_{t}^{2}\left(\frac{\partial \tau}{\partial z}\right)^{2}+a_{1} v_{t} \frac{\partial \tau}{\partial z}+a_{0}=0
$$

where

$$
\begin{aligned}
& a_{0}=2 v^{2} v_{t}^{2} \eta \sin ^{2} \theta \cos ^{2} \theta\left(\cos \phi \frac{\partial \tau}{\partial x}-\sin \phi \frac{\partial \tau}{\partial y}\right)^{4}-v^{2}(2 \eta+1) \cos ^{2} \theta\left(\cos \phi \frac{\partial \tau}{\partial x}-\sin \phi \frac{\partial \tau}{\partial y}\right)^{2} \\
& +2 v^{2} v_{t}^{2} \eta \sin ^{2} \theta\left(\sin \phi \frac{\partial \tau}{\partial x}+\cos \phi \frac{\partial \tau}{\partial y}\right)^{2}\left(\cos \phi \frac{\partial \tau}{\partial x}-\sin \phi \frac{\partial \tau}{\partial y}\right)^{2} \\
& -v^{2}(2 \eta+1)\left(\sin \phi \frac{\partial \tau}{\partial x}+\cos \phi \frac{\partial \tau}{\partial y}\right)^{2}-v_{t}^{2} \sin ^{2} \theta\left(\cos \phi \frac{\partial \tau}{\partial x}-\sin \phi \frac{\partial \tau}{\partial y}\right)^{2}+1, \\
& a_{1}=-\frac{2}{v_{t}} \sin \theta \cos \theta\left(\cos \phi \frac{\partial \tau}{\partial x}-\sin \phi \frac{\partial \tau}{\partial y}\right)\left(v _ { t } \left(2 v ^ { 2 } \eta \left(\sin ^{2} \theta \cos \phi \frac{\partial \tau}{\partial x}\left(2 v_{t} \sin \phi \frac{\partial \tau}{\partial y}-1\right)\right.\right.\right. \\
& +v_{t}\left(\frac{\partial \tau}{\partial x}\right)^{2}\left(\cos ^{2} \theta \cos ^{2} \phi+\sin ^{2}(\phi)\right)+\frac{\partial \tau}{\partial y}\left(v_{t} \frac{\partial \tau}{\partial y}\left(\cos ^{2} \theta \sin ^{2} \phi+\cos ^{2}(\phi)\right)\right. \\
& \left.\left.\left.\left.+\sin ^{2} \theta \sin \phi\right)\right)-v_{t}\right)+v^{2}(2 \eta+1)\right), \\
& a_{2}=\frac{1}{4}\left(v ^ { 2 } \eta \left(-4 \sin ^{2} \theta(3 \cos (2 \theta)+2) \cos (2 \phi)\left(\left(\frac{\partial \tau}{\partial x}\right)^{2}-\left(\frac{\partial \tau}{\partial y}\right)^{2}\right)+8 \sin ^{2} \theta(3 \cos (2 \theta)+2)\right.\right. \\
& \left.\left.\sin (2 \phi) \frac{\partial \tau}{\partial x} \frac{\partial \tau}{\partial y}+(2 \cos (2 \theta)+3 \cos (4 \theta)+3)\left(\left(\frac{\partial \tau}{\partial x}\right)^{2}+\left(\frac{\partial \tau}{\partial y}\right)^{2}\right)\right)-4 \cos ^{2} \theta\right) \\
& -\frac{v^{2}(2 \eta+1) \sin ^{2} \theta}{v_{t}^{2}} \\
& a_{3}=\frac{v^{2} \eta \sin (4 \theta)\left(\cos \phi \frac{\partial \tau}{\partial x}-\sin \phi \frac{\partial \tau}{\partial y}\right)}{v_{t}}, \\
& a_{4}=\frac{v^{2} \eta \sin ^{2} \theta \cos ^{2} \theta}{v_{t}^{2}}
\end{aligned}
$$

To develop equations for the coefficients of a traveltime expansion in 3D from a background elliptical anisotropy with a vertical symmetry axis I use vector notations $\left(n_{x}\right.$ and $\left.n_{y}\right)$ to describe the tilt angles, where the components of this $2 \mathrm{D}$ vector describe the projection of the symmetry axis on each of the $x-z$ and $y-z$ planes, respectively. As a result,

$$
n_{x}=\sin \theta \cos \phi
$$

and

$$
n_{y}=\sin \theta \sin \phi .
$$


Using these two equations to solve for $\sin \theta$ and $\sin \phi$ and plugging them into equation D-1 yields an eikonal for TTI media in terms of $n_{x}$ and $n_{y}$. Thus, inserting the following trial solution

$\tau(x, y, z) \approx \tau_{0}(x, y, z)+\tau_{\eta}(x, y, z) \eta+\tau_{\eta_{2}}(x, y, z) \eta^{2}+\tau_{n_{x}}(x, y, z) n_{x}+\tau_{n_{y}}(x, y, z) n_{\text {女( }}$ D-9)

where $\eta, n_{x}$, and $n_{y}$ are independent parameters and small, into the eikonal equation yields an extremely long equation. Again, setting the coefficients of the independent parameters $\left(\eta, n_{x}\right.$, and $\left.n_{y}\right)$ to zero in the equation gives the eikonal equation for elliptical anisotropy with vertical symmetry axis. On the other hand, the coefficients of the first power of the independent parameters yield:

$$
\begin{aligned}
v^{2} \frac{\partial \tau_{0}}{\partial x} \frac{\partial \tau_{\eta}}{\partial x}+v^{2} \frac{\partial \tau_{0}}{\partial y} \frac{\partial \tau_{\eta}}{\partial y}+v_{t}^{2} \frac{\partial \tau_{0}}{\partial z} \frac{\partial \tau_{\eta}}{\partial z} & =v^{2}\left(\left(v_{t}^{2}\left(\frac{\partial \tau_{0}}{\partial z}\right)^{2}-1\right)\left(\left(\frac{\partial \tau_{0}}{\partial x}\right)^{2}+\left(\frac{\partial \tau_{0}}{\partial y}\right)^{2}\right)\right), \\
v^{2} \frac{\partial \tau_{0}}{\partial y} \frac{\partial \tau_{n_{x}}}{\partial y}+v^{2} \frac{\partial \tau_{0}}{\partial x} \frac{\partial \tau_{n_{x}}}{\partial x}+v_{t}^{2} \frac{\partial \tau_{0}}{\partial z} \frac{\partial \tau_{n_{x}}}{\partial z} & =-\left(v^{2}-v_{t}^{2}\right) \frac{\partial \tau_{0}}{\partial x} \frac{\partial \tau_{0}}{\partial z}, \\
v^{2} \frac{\partial \tau_{0}}{\partial y} \frac{\partial \tau_{n_{y}}}{\partial y}+v^{2} \frac{\partial \tau_{0}}{\partial x} \frac{\partial \tau_{n_{y}}}{\partial x}+v_{t}^{2} \frac{\partial \tau_{0}}{\partial z} \frac{\partial \tau_{n_{y}}}{\partial z} & =-\left(v^{2}-v_{t}^{2}\right) \frac{\partial \tau_{0}}{\partial y} \frac{\partial \tau_{0}}{\partial z},
\end{aligned}
$$

corresponding to $\eta, n_{x}$, and $n_{y}$, respectively.

The coefficient of the $\eta^{2}$ term, for higher accuracy in $\eta$, is given by

$$
\begin{gathered}
2 v^{2} \frac{\partial \tau_{0}}{\partial x} \frac{\partial \tau_{\eta_{2}}}{\partial x}+2 v^{2} \frac{\partial \tau_{0}}{\partial y} \frac{\partial \tau_{\eta_{2}}}{\partial y}+2 v_{t}^{2} \frac{\partial \tau_{0}}{\partial z} \frac{\partial \tau_{\eta_{2}}}{\partial z}=4 v_{t}^{2} v^{2}\left(\frac{\partial \tau_{0}}{\partial z}\right)^{2}\left(\frac{\partial \tau_{0}}{\partial x} \frac{\partial \tau_{\eta}}{\partial x}+\frac{\partial \tau_{0}}{\partial y} \frac{\partial \tau_{\eta}}{\partial y}\right) \\
+4 v_{t}^{2} v^{2} \frac{\partial \tau_{0}}{\partial z}\left(\left(\frac{\partial \tau_{0}}{\partial x}\right)^{2}+\left(\frac{\partial \tau_{0}}{\partial y}\right)^{2}\right) \frac{\partial \tau_{\eta}}{\partial z}-v^{2}\left(\left(\frac{\partial \tau_{\eta}}{\partial x}\right)^{2}+\left(\frac{\partial \tau_{\eta}}{\partial y}\right)^{2}\right)-4 v^{2}\left(\frac{\partial \tau_{0}}{\partial x} \frac{\partial \tau_{\eta}}{\partial x}+\frac{\partial \tau_{0}}{\partial y} \frac{\partial \tau_{\eta}}{\partial y}\right) \\
-v_{t}^{2}\left(\frac{\partial \tau_{\eta}}{\partial z}\right)^{2} .
\end{gathered}
$$

These first-order PDEs, when solved, provide traveltime approximations using equation D-9 for 3D TI media in a generally inhomogeneous elliptical anisotropic background.

For a homogeneous medium simplification, the traveltime is given by the following analytical relation in 3-D elliptical anisotropic media:

$$
\tau_{0}(x, y, z)=\sqrt{\frac{x^{2}+y^{2}}{v^{2}}+\frac{z^{2}}{v_{t}^{2}}},
$$

which satisfies the eikonal equation B-2 in 3D. Using equation D-12, I evaluate $\frac{\partial \tau_{0}}{\partial x}, \frac{\partial \tau_{0}}{\partial y}$ and $\frac{\partial \tau_{0}}{\partial z}$ and insert them into equations D-10 to solve these first-order linear equations to obtain

$$
\tau_{\eta}(x, y, z)=-\frac{v_{t}^{4}\left(x^{2}+y^{2}\right)^{2} \sqrt{\frac{x^{2}+y^{2}}{v^{2}}+\frac{z^{2}}{v_{t}^{2}}}}{\left(v^{2} z^{2}+v_{t}^{2}\left(x^{2}+y^{2}\right)\right)^{2}},
$$




$$
\begin{aligned}
\tau_{n_{x}}(x, y, z) & =\frac{\left(v_{t}^{2}-v^{2}\right) x z}{v^{2} v_{t}^{2} \sqrt{\frac{x^{2}+y^{2}}{v^{2}}+\frac{z^{2}}{v_{t}^{2}}}}, \\
\tau_{n_{y}}(x, y, z) & =\frac{\left(v_{t}^{2}-v^{2}\right) y z}{v^{2} v_{t}^{2} \sqrt{\frac{x^{2}+y^{2}}{v^{2}}+\frac{z^{2}}{v_{t}^{2}}}},
\end{aligned}
$$

respectively.

I now evaluate $\frac{\partial \tau_{\eta}}{\partial x}, \frac{\partial \tau_{\eta}}{\partial y}$, and $\frac{\partial \tau_{\eta}}{\partial z}$ and use them to solve equation D-11. After some tedious algebra, I obtain

$$
\tau_{\eta_{2}}(x, y, z)=\frac{3 v_{t}^{6}\left(x^{2}+y^{2}\right)^{3} \sqrt{\frac{x^{2}+y^{2}}{v^{2}}+\frac{z^{2}}{v_{t}^{2}}}\left(4 v^{2} z^{2}+v_{t}^{2}\left(x^{2}+y^{2}\right)\right)}{2\left(v^{2} z^{2}+v_{t}^{2}\left(x^{2}+y^{2}\right)\right)^{4}} .
$$

The application of Pade approximation on the expansion in $\eta$, by finding a first order polynomial representation in the denominator, yields a TI equation that is accurate for large $\eta$ (Alkhalifah, 2010), as well as small tilt, given by

$$
\begin{aligned}
\tau(x, y, z) \approx & \frac{1}{v^{2} v_{t}^{2} \sqrt{\frac{x^{2}+y^{2}}{v^{2}}+\frac{z^{2}}{v_{t}^{2}}}\left(2 v^{4} z^{4}+4 v^{2} v_{t}^{2} z^{2}(3 \eta+1)\left(x^{2}+y^{2}\right)+v_{t}^{4}(3 \eta+2)\left(x^{2}+y^{2}\right)^{2}\right)} \\
& \left(2 v^{6} z^{5}(z-\sin \theta(x \cos \phi+y \sin \phi))-2 v_{t}^{2} v^{4} z^{3}\left(\left((6 \eta+2)\left(x^{2}+y^{2}\right)-z^{2}\right)\right.\right. \\
& \left.(\sin \theta(x \cos \phi+y \sin \phi))-3 z(2 \eta+1)\left(x^{2}+y^{2}\right)\right)-v_{t}^{4} v^{2} z\left(x^{2}+y^{2}\right) \\
& \left(\left((3 \eta+2)\left(x^{2}+y^{2}\right)-4 z^{2}(3 \eta+1)\right) \sin \theta(x \cos \phi+y \sin \phi)-z(13 \eta+6)\left(x^{2}+y^{2}\right)\right) \\
& \left.+v_{t}^{6}\left(x^{2}+y^{2}\right)^{2}\left(x^{2}(\eta+2)+z(3 \eta+2) \sin \theta(x \cos \phi+y \sin \phi)+y^{2}(\eta+2)\right)\right) .(\mathrm{D}-15)
\end{aligned}
$$

